Anaesthesist 2014 - 63:62

DOI 10.1007/s00101-013-2281-8

Online publiziert: 19. Januar 2014

(c) Springer-Verlag Berlin Heidelberg 2014

E. Schneck · S. Schaumberg · C. Koch · M. Rickert · C. Lichtenstern

Klinik für Anaesthesiologie und Operative Intensivmedizin,

Universitätsklinikum Gießen und Marburg, Gießen

\title{
Erratum zu: Anästhesiologisches Management des Gitelman-Syndroms
}

\section{Anaesthesist (2013) 62:728-733}

Die Autoren machen darauf aufmerksam, dass in diesem Beitrag 2 Werte in - Tab. 1 vertauscht wurden. Die korrigierten Werte sind fett dargestellt.

\section{Korrespondenzadresse}

\section{Dr. C. Lichtenstern}

Klinik für Anaesthesiologie und Operative Intensivmedizin, Universitätsklinikum Gießen und Marburg

Rudolf-Buchheim Str. 7, 35392 Gießen

christoph.lichtenstern@

chiru.med.uni-giessen.de
Die Online-Version des Originalartikels können Sie unter http://dx.doi.org/10.1007/s00101-0132218-2 finden.

Tab. 1 Darstellung der Blutgasanalysen

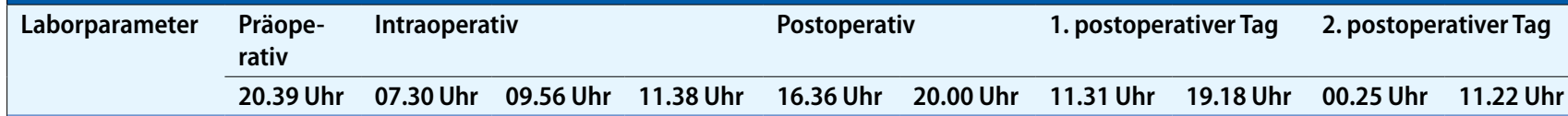

\section{Blutgase (peri-}

pher venös)

\begin{tabular}{|c|c|c|c|c|c|c|c|c|c|c|}
\hline $\mathrm{pH}$ & & & 7,344 & 7,371 & 7,31 & 7,35 & 7,418 & 7,407 & & 7,40 \\
\hline $\mathrm{pCO}_{2}(\mathrm{mmHg})$ & & & 45,3 & 38,4 & 44,5 & 35,8 & 33,2 & 36,3 & & 36,1 \\
\hline $\mathrm{pO}_{2}(\mathrm{mmHg})$ & & & 51,6 & 59,8 & 72,0 & 89,0 & 60,4 & 64,1 & & 51,3 \\
\hline $\mathrm{SO}_{2}(\%)$ & & & 81,7 & 91,2 & 92,3 & 96,5 & 90,5 & 91,1 & & 84,0 \\
\hline $\begin{array}{l}\text { "Base excess" } \\
(\mathrm{mmol} / \mathrm{l})\end{array}$ & & & $-1,1$ & $-2,0$ & $-3,7$ & $-5,2$ & $-2,6$ & $-1,5$ & & $-2,0$ \\
\hline $\begin{array}{l}\text { Standardbikarbo- } \\
\text { nat }(\mathrm{mmol} / \mathrm{l})\end{array}$ & & & 23,4 & 22,5 & 21,3 & 20,1 & & & & \\
\hline \multicolumn{11}{|l|}{ Elektrolyte } \\
\hline Natrium $(\mathrm{mmol} / \mathrm{l})$ & 134 & 137 & 137 & 136 & 136 & 132 & 135 & 137 & & 137 \\
\hline Kalium (mmol/l) & 4,6 & 3,9 & 4,23 & 4,75 & 4,9 & 4,5 & 3,53 & 4,37 & & 3,83 \\
\hline Kalzium (mmol/l) & 1,9 & 1,8 & 1,03 & 1,09 & 1,8 & 1,8 & 0,96 & 0,95 & & 0,99 \\
\hline $\begin{array}{l}\text { Magnesium } \\
(\mathrm{mmol} / \mathrm{l})\end{array}$ & 2,61 & 2,57 & 1,11 & 1,46 & 3,99 & 3,03 & 2,57 & 2,67 & 2,61 & 2,78 \\
\hline \multicolumn{11}{|l|}{$\begin{array}{l}\text { Retentionspara- } \\
\text { meter }\end{array}$} \\
\hline Kreatinin (mg/dl) & 1,2 & & & & 1,0 & & 0,9 & 1,0 & 1,0 & 1,0 \\
\hline Harnstoff (mg/dl) & 25 & & & & 24 & & 27 & 22 & 23 & 39 \\
\hline
\end{tabular}

